Note

Subscriber access provided by NEW YORK UNIV

\title{
Cycloisomerization of Conjugated Trienones and Isomeric 2H-Pyrans: Unified Strategy toward Cyclopenta[b]furans
}

Martin Jorge Riveira, Gaston Nicolas Quiroga, Ernesto G. Mata, Vincent Gandon, and Mirta P. Mischne J. Org. Chem., Just Accepted Manuscript • DOI: 10.1021/acs.joc.5b00818 • Publication Date (Web): 27 May 2015

Downloaded from http://pubs.acs.org on May 29, 2015

\section{Just Accepted}

"Just Accepted" manuscripts have been peer-reviewed and accepted for publication. They are posted online prior to technical editing, formatting for publication and author proofing. The American Chemical Society provides "Just Accepted" as a free service to the research community to expedite the dissemination of scientific material as soon as possible after acceptance. "Just Accepted" manuscripts appear in full in PDF format accompanied by an HTML abstract. "Just Accepted" manuscripts have been fully peer reviewed, but should not be considered the official version of record. They are accessible to all readers and citable by the Digital Object Identifier (DOI囚). "Just Accepted" is an optional service offered to authors. Therefore, the "Just Accepted" Web site may not include all articles that will be published in the journal. After a manuscript is technically edited and formatted, it will be removed from the "Just Accepted" Web site and published as an ASAP article. Note that technical editing may introduce minor changes to the manuscript text and/or graphics which could affect content, and all legal disclaimers and ethical guidelines that apply to the journal pertain. ACS cannot be held responsible for errors or consequences arising from the use of information contained in these "Just Accepted" manuscripts.

\section{ACS Publications}




\title{
Cycloisomerization of Conjugated Trienones and Isomeric 2H-Pyrans: Unified Strategy toward Cyclopenta[b]furans
}

\author{
Martín J. Riveira, ${ }^{\dagger}$ Gastón N. Quiroga, ${ }^{\dagger}$ Ernesto G. Mata, ${ }^{\dagger}$ Vincent Gandon ${ }^{*, \ddagger, \neq}$, and Mirta \\ P. Mischne $e^{* \dagger}$ \\ ${ }^{\dagger}$ Instituto de Química Rosario-CONICET, Facultad de Ciencias Bioquímicas y \\ Farmacéuticas, Universidad Nacional de Rosario, Suipacha 531, 2000 Rosario, Santa Fe, \\ Argentina. \\ mischne@iquir-conicet.gov.ar \\ ${ }^{\ddagger}$ ICMMO (UMR CNRS 8182), Université Paris-sud 11, 91405 Orsay Cedex, France. \\ ${ }^{\ddagger}$ Institut de Chimie des Substances Naturelles, CNRS, Avenue de la Terrasse, 91198 Gif- \\ sur-Yvette Cedex, France. \\ vincent.gandon@u-psud.fr
}

\section{TABLE OF CONTENTS/ABSTRACT GRAPHIC}

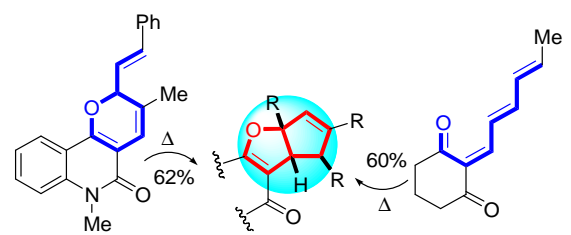

\begin{abstract}
Conjugated trienones and isomeric $2 H$-pyrans were found to engage in a novel cycloisomerization cascade toward cyclopenta[b]furan derivatives. Knoevenagel chemistry and pericyclic reactions meet again to expand the polyene-carbonyl manifold.
\end{abstract}


Cycloisomerization reactions do not cease to amaze chemists who stand still trying to grasp the circle dance of atoms in their molecules. Indeed Nature also makes use of these atom-economical transformations to construct many of the rings found embedded in its precious products. One notable example of this is the pericyclic oxa- $6 \pi$-electrocyclization reaction of dienone (or dienal) structures to yield the well-known $2 \mathrm{H}$-pyran isomers. This reversible process is usually masked by subsequent transformations, mostly [4+2] cycloadditions, accounting for the biosynthesis of xanthipungolide, torreyanic acid, epoxyquinols A-C, pinnatal, and so many other elegant natural molecules of interest. ${ }^{1-3}$

The domino Knoevenagel condensation/oxa- $6 \pi$-electrocyclization reaction between enals and 1,3-dicarbonyl substrates, usually referred to as formal [3+3] cycloaddition, has become a very useful strategy for the synthesis of natural $2 H$-pyran-containing compounds. $^{4,5}$ Our studies on this condensation using $\alpha, \beta, \gamma, \delta$-unsaturated aldehydes revealed that, depending on the substitution pattern and electronic properties, apart from the expected $\pi$-conjugated dicarbonyl products and their $2 H$-pyran isomers ( $\mathbf{1}$ and 2 ), previously unrelated cyclopenta[b]furan isomers of type $\mathbf{3}$ can also be obtained (Scheme 1). ${ }^{6}$ To account for the formation of these unexpected products, a domino Knoevenagel/cationic bicyclization pathway was proposed featuring a pentadienylcyclopentenyl cation rearrangement at the end of the polyene chain of the putative, nonisolable, trienone intermediates. Indeed, iron(III) chloride was shown to catalyze the isomerization of stable isolable trienones of type $\mathbf{1}$ into these heterocyclic isomers $(\mathbf{3})$ and the process was hence classified as an interrupted vinylogous iso-Nazarov reaction. ${ }^{7}$ 


\section{Scheme 1. Manifold of the Knoevenagel chemistry using 1,3-dicarbonyl substrates and} $\alpha, \beta, \gamma, \delta$-unsaturated aldehydes

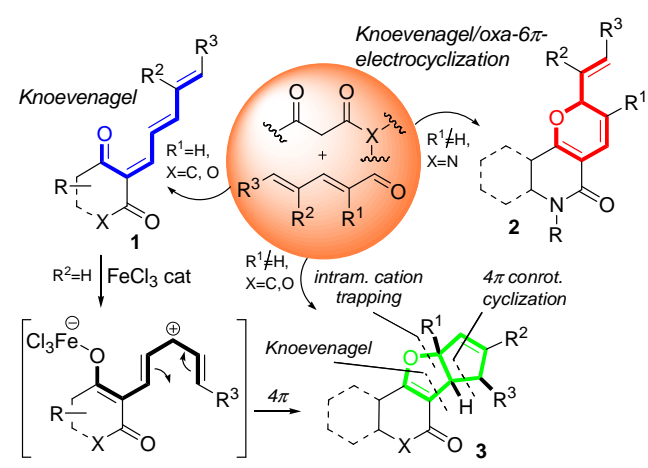

Due to the wide application of molecular switches in the fabrication of novel materials and devices, the dienone $/ 2 \mathrm{H}$-pyran isomerism has received considerable attention. ${ }^{8,9}$ Krasnaya and Hsung have both independently provided examples of dienones that rearrange to their pyranic isomers upon thermal treatment. ${ }^{10,11}$ In order to test whether vinylogous systems of type $\mathbf{1}$ behave in such a way, a toluene solution of model substrate 1a, prepared via Knoevenagel condensation between 1,3-cyclohexanedione and sorbaldehyde, was heated under reflux (Scheme 2A). To our surprise, after 30 hours of heating, cyclopenta[b]furan isomer 3a was found as the only isolable product of the reaction. The same reactivity profile was found for other related substrates (Scheme 2B). Although the use of higher-boiling solvents or sealed systems provided faster transformations, marked reduced yields ensued. The possibility to incorporate the Knoevenagel condensation and cycloisomerization process into a one-pot transformation was explored as well but, unfortunately, no suitable experimental conditions for making it a valuable process could be found. 
Although only moderate yields could be obtained and indeed more efficient transformations were found using our previously developed acid-promoted approach to these cyclopenta $[b]$ furan derivatives; ${ }^{7,12}$ from a mechanistic viewpoint we were completely amazed by this notable cycloisomerization. To account for cyclopenta[b]furan formation, a mechanistic proposal was elaborated indeed involving originally sought $2 \mathrm{H}$-pyran isomers as intermediates (Scheme 2C). An initial oxa-6 $\pi$-electrocyclic ring closure/opening sequence, similar to that proposed to account for the isomerization of retinals, ${ }^{13}$ would provide reactive $\gamma, \delta$-Z-diastereomer of trienone 1a substrate. Strain could then force polyene chain to a conformation in which it is dissected into two $4 \pi \mathrm{e}^{-}$moieties. Intramolecular $\left[{ }_{\pi} 4_{\mathrm{a}}+{ }_{\pi} 4_{\mathrm{s}}\right]$ cycloaddition would then afford cyclopenta[$[b]$ furan product $3 \mathbf{3 a} .{ }^{1} \mathrm{H}$ NMR monitoring of these reactions carried out in toluene- $d_{8}$ at $100{ }^{\circ} \mathrm{C}$ threw no evidence of any of the proposed intermediates. DFT calculations at the M06/6-31+G** level of theory were then performed to assess the feasibility of the proposed pathway (Scheme 3 and Supporting Information; for other DFT functionals also see Supporting Information). ${ }^{14}$ Overall, the bicyclization of substrate 1a into cyclopenta[b]furan isomer 3a takes six elementary steps and liberates $7.8 \mathrm{kcal} / \mathrm{mol}$ of Gibbs free energy: (i) conversion of $s$ trans/s-trans E/E 1a into the $s$-cis/s-trans E/E isomer $\mathbf{A}$; (ii) oxa-6 6 electrocyclization to give $\mathbf{2 a}$; (iii) retro oxa-6 $\pi$ leading to the $s$-cis/s-trans $Z / E$ isomer $\mathbf{B}$; (iv) conversion of $\mathbf{B}$ into the $s$-trans/s-trans $Z / E$ isomer $\mathbf{C}$; (v) formation of the $s$-trans/s-cis Z/E isomer $\mathbf{D}$; and (vi) $\left[\pi 4_{\mathrm{a}}+\pi 4_{\mathrm{s}}\right]$ cycloaddition giving rise to the final product 3a. As shown by the lengths of the forming $\mathrm{C}-\mathrm{O}$ and $\mathrm{C}-\mathrm{C}$ bonds in TS D-3a (2.68 $\AA$ and $1.94 \AA$ respectively), the construction of the two rings occurs in an asynchronous fashion in favor of the 
cyclopentene framework. This last step is the rate determining one $\left(\Delta G^{\ddagger}{ }_{298}=28.1\right.$ $\mathrm{kcal} / \mathrm{mol})$.

Scheme 2. Thermal isomerization of conjugated trienones 1 to cyclopenta[b]furan derivatives 3

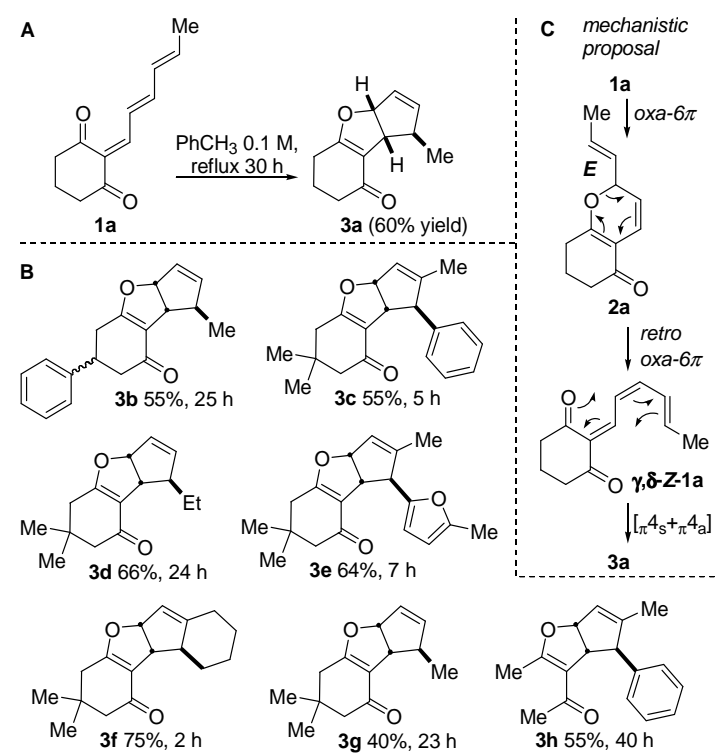

Scheme 3. Calculated energy profile $\left(\Delta G_{298}, \mathrm{kcal} / \mathrm{mol}\right)$ for the bicyclization of conjugated trienone 1a

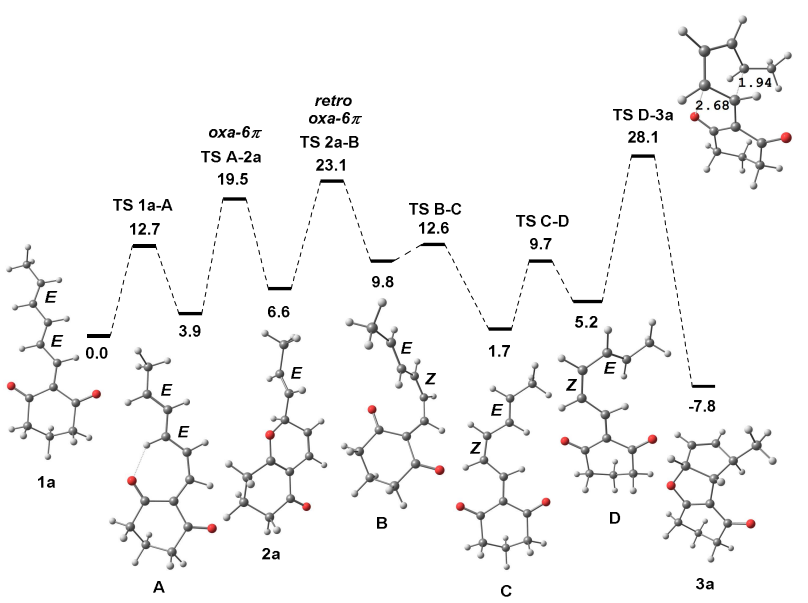


Our unexpected findings made us revisit the original tandem Knoevenagel/polycyclization reaction between $\beta$-diketones or $\beta$-ketoesters and $\alpha$ substituted dienals, for which conjugated trienones were proposed as direct precursors of the cyclopenta[b]furan products $\mathbf{3}$, and $2 \mathrm{H}$-pyrans of type $\mathbf{2}$ were regarded rather as products of an unrelated dead-end pathway. ${ }^{6 a}$ In order to find evidence of any intermediate that could be involved, condensation between model substrates dimedone (4) and 2-methyl5-phenyl-penta-2,4-dienal (5) was carried out in an NMR tube (Figure 1).

Figure 1. ${ }^{1} \mathrm{H}$ NMR monitoring of the tandem Knoevenagel-bicylization sequence toward cyclopenta[b]furans (EDDA = ethylenediammonium diacetate)
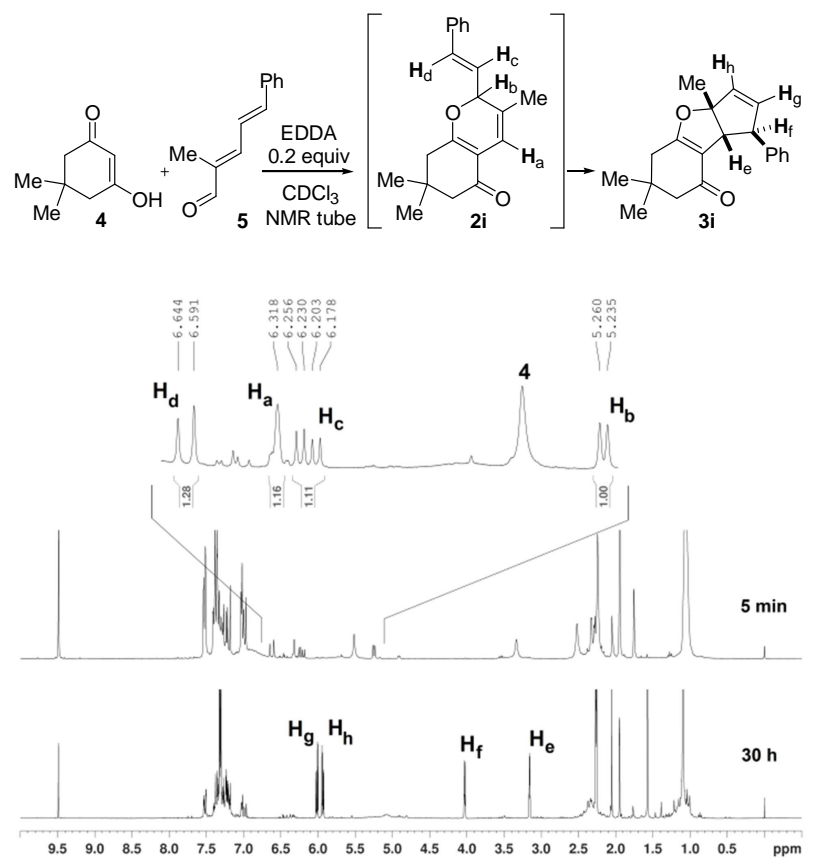

As soon as reaction commenced and before any product $\mathbf{3 i}$ was produced, ${ }^{1} \mathrm{H}$ NMR monitoring indeed revealed the presence of a molecular species that was gradually consumed and completely depleted after 30 hours (Figure 1). This presumable intermediate 
gives rise to signals which can be tentatively attributed to the structure of $2 \mathrm{H}$-pyran $2 \mathbf{i}$. The uncrowded vinyl region of the ${ }^{1} \mathrm{H}$ NMR spectrum features a broad singlet at $6.32 \mathrm{ppm}\left(\mathrm{H}_{\mathrm{a}}\right)$, two doublets centered at 6.62 and $5.25 \mathrm{ppm}\left(\mathrm{H}_{\mathrm{d}}\right.$ and $\mathrm{H}_{\mathrm{b}}, J=16.0$ and $7.5 \mathrm{~Hz}$, respectively) and a doublet of doublets at $6.22 \mathrm{ppm}\left(\mathrm{H}_{\mathrm{c}}, J=16.0,7.5 \mathrm{~Hz}\right)$. In addition, the oxygen bearing methine carbon would account for the ${ }^{13} \mathrm{C}$ NMR signal at $81.1 \mathrm{ppm}$ (see Supporting Information). Since all attempts to isolate this intermediate foundered, possibly due to rapid isomerization to product $\mathbf{3 i}$, we envisioned that hydrogenation would allow its trapping. ${ }^{15}$ After considerable experimentation using different aldehydes and dicarbonyl substrates, pyran $2 \mathbf{j}_{\text {red }}$ could be isolated in low yield along with expected reduced cyclopenta $[b]$ furan $\mathbf{3} \mathbf{j}_{\text {red }}$ when reaction between dimedone (4) and 2,5-diphenyl-penta-2,4-dienal (6) was interrupted before completion and submitted to hydrogenation under heterogeneous conditions using Adam's catalyst (Scheme 4).

\section{Scheme 4. Further evidence for the intermediacy of $2 \mathrm{H}$-pyrans en route to cyclopenta[b]furan isomers}

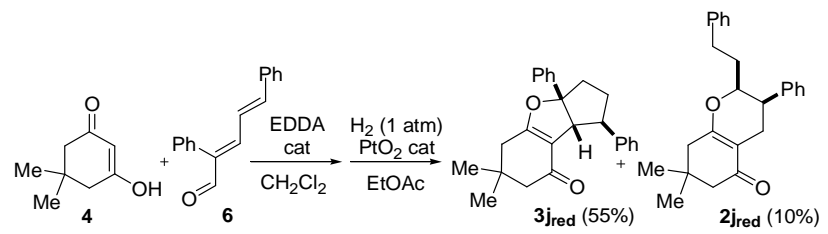

This finding that $2 H$-pyrans could be transient intermediates en route to cyclopenta[b]furans prompted us to evaluate whether the cycloisomerization of stable, isolable $2 \mathrm{H}$-pyrans, may be feasible. As a proof-of-concept, compound $\mathbf{2} \mathbf{k}$ was chosen as model substrate (Scheme 5). This $2 \mathrm{H}$-pyran was prepared via Knoevenagel condensation between aldehyde 5 and 4-hydroxy-1-methyl-2(1H)-quinolone (7) as previously reported. ${ }^{6 \mathrm{a}}$ 
When a toluene solution of $\mathbf{2 k}$ was subjected to reflux conditions, cyclopenta[b]furoquinolone $3 \mathbf{k}$ was indeed found as the only isolable product in $62 \%$ yield (Scheme 5). In addition, the one-pot Knoevenagel condensation/cycloisomerization sequence, in this case, was shown to be accessible ( $43 \%$ yield). The isomerization of pyran 2k could also be promoted using ferric chloride (1 equiv) in dichloromethane at reflux, albeit in lower yield (40\%). In this case, the reaction would proceed through initial Lewis acid promoted heterolytic C-O bond cleavage generating key polyenyl cation $\mathbf{E}$ which, after a $4 \pi \mathrm{e}^{-}$conrotatory electrocyclization and subsequent stereoselective trapping of brand new cyclopentenyl cation intermediate by enolic oxygen, would provide cis-fused cyclopenta[b]furan product $\mathbf{3 k}$.

\section{Scheme 5. Rearrangement of a stable 2-vinyl-2H-pyran}

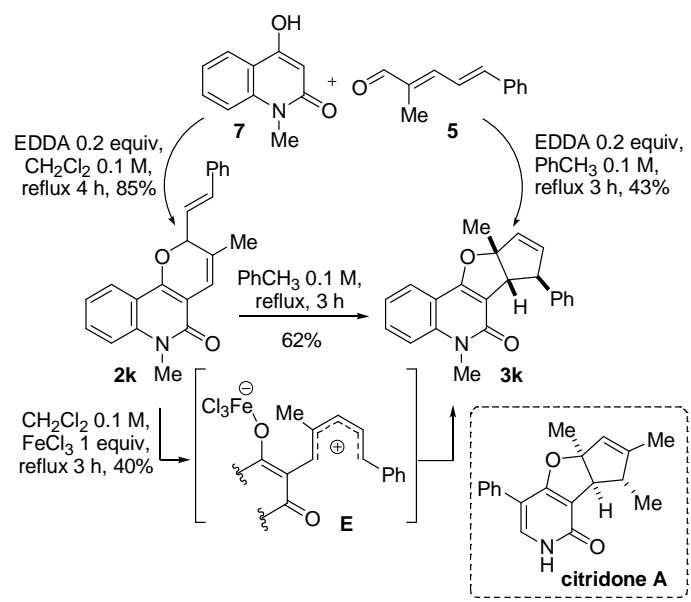

In summary, a novel cycloisomerization pathway viable for Knoevenagel chemistry was disclosed. Trienones and $2 \mathrm{H}$-pyrans were found to smoothly rearrange to previously unrelated isomers, cyclopenta[b]furans. Indeed, this heterocyclic scaffold is widespread in Nature. ${ }^{16}$ Taking into account the ease with which these assemblies are stereoselectively 
constructed from simple precursors, the question raises as to whether this approach could account for the biosynthetic origin of natural products such as citridone A (Scheme 5), ${ }^{17}$ which shares the same tricyclic cyclopentafuropyridone core present in product $\mathbf{3 k}$. Efforts to clarify this scenario are underway.

\section{EXPERIMENTAL SECTION}

Unsaturated aldehydes $\mathbf{5}$ and $\mathbf{6}$ have been previously prepared by our group. ${ }^{6}$

General procedure for the preparation of substrates 1 and 2 via condensation of 1,3dicarbonyl compounds and unsaturated aldehydes. A mixture of 1,3-dicarbonyl compound (1 mmol), unsaturated aldehyde (1 mmol, 1 equiv) and 1,2ethylenediammonium diacetate (EDDA, $36 \mathrm{mg}, 0.2 \mathrm{mmol})$ in $\mathrm{CH}_{2} \mathrm{Cl}_{2}(5.0 \mathrm{ml}, 0.2 \mathrm{M}$ ) was heated at reflux until complete consumption of aldehyde substrate (TLC monitoring, approximately 3-4 hours). The solvent was then evaporated under reduced pressure and the residue was purified by flash column chromatography on silica gel (eluent hexanes/ethyl acetate) to afford the desired unsaturated substrates $\mathbf{1}$ or $\mathbf{2}$. Conjugated dicarbonyl substrates 1a-h and $2 H$-pyran substrate $2 \mathbf{k}$ have been previously prepared by our group using this protocol. ${ }^{6,7}$ For the ${ }^{1} \mathrm{H}$ NMR monitoring of the tandem Knoevenagel-bicylization sequence between dicarbonyl substrate $\mathbf{4}$ and unsaturated aldehyde $\mathbf{5}$ toward cyclopenta[b]furan $3 \mathbf{3 i}^{6 \mathrm{~b}}$, this same protocol was used employing $\mathrm{CDCl}_{3}$ as solvent instead of $\mathrm{CH}_{2} \mathrm{Cl}_{2}$.

General procedure for the thermal cycloisomerization toward cyclopenta[b]furans 3 . A solution of conjugated dicarbonyl substrate 1 or $2 H$-pyran $2(1 \mathrm{mmol})$ in toluene $(10.0$ $\mathrm{ml}, 0.1 \mathrm{M}$ ) was heated at reflux until complete consumption of substrate (TLC monitoring). The solvent was then evaporated under reduced pressure and the crude product was purified 
by flash column chromatography on silica gel (eluent hexanes/ethyl acetate) to afford the following compounds.

Cyclopenta[b]furan $3 a^{7}$ Yield: $114 \mathrm{mg}(60 \%)$. Colorless to pale yellow solid. mp 46.0$47.0{ }^{\circ} \mathrm{C} .{ }^{1} \mathrm{H}$ NMR $\left(300 \mathrm{MHz}, \mathrm{CDCl}_{3}\right): \delta 6.02(\mathrm{dd}, J=5.6 \mathrm{~Hz}, J=2.2 \mathrm{~Hz}, 1 \mathrm{H}), 5.82(\mathrm{dt}, J=$ $8.6 \mathrm{~Hz}, J=1.8 \mathrm{~Hz}, 1 \mathrm{H}), 5.74(\mathrm{dt}, J=5.6 \mathrm{~Hz}, J=2.0 \mathrm{~Hz}, 1 \mathrm{H}), 3.27(\mathrm{dq}, J=8.5 \mathrm{~Hz}, J=1.8$ $\mathrm{Hz}, 1 \mathrm{H}$ ), 2.84 (qquint, $J=7.3 \mathrm{~Hz}, J=1.9 \mathrm{~Hz}, 1 \mathrm{H}$ ), $2.39(\mathrm{td}, J=6.4 \mathrm{~Hz}, J=1.6 \mathrm{~Hz}, 2 \mathrm{H}$ ), $2.32(\mathrm{t}, J=6.3 \mathrm{~Hz}, 2 \mathrm{H}), 2.00$ (quint, $J=6.4 \mathrm{~Hz}, 2 \mathrm{H}), 1.18(\mathrm{~d}, J=7.3 \mathrm{~Hz}, 3 \mathrm{H})$.

Cyclopenta[b]furans $3 \boldsymbol{b}^{7}{ }^{7}$ Yield $147 \mathrm{mg}$ (55\%). Colorless solid. mp 70.0-71.0 ${ }^{\circ} \mathrm{C} .{ }^{1} \mathrm{H}$ NMR (300 MHz, $\mathrm{CDCl}_{3}$ ): $\delta$ 7.37-7.29 (overlapping m, 4H), 7.28-7.20 (overlapping m, 6H), 6.086.02 (overlapping m, 2H), 5.92-5.84 (overlapping m, 2H), 5.80-5.72 (overlapping m, 2H), 3.47-3.27 (overlapping m, 4H), 2.95-2.83 (overlapping m, 2H), 2.67-2.54 (overlapping m, 8H), 1.23-1.17 (overlapping m, 6H).

Cyclopenta[b]furan 3c. ${ }^{6 a}$ Yield $162 \mathrm{mg}$ (55\%). Pale yellow solid. mp 95.0-96.0 ${ }^{\circ} \mathrm{C} .{ }^{1} \mathrm{H}$ NMR (300 MHz, $\left.\mathrm{CDCl}_{3}\right): \delta 7.34-7.27(\mathrm{~m}, 2 \mathrm{H}), 7.23-7.17(\mathrm{~m}, 3 \mathrm{H}), 5.90(\mathrm{dm}, J=8.5 \mathrm{~Hz}$, $1 \mathrm{H}), 5.67(\mathrm{q}, J=1.6 \mathrm{~Hz}, 1 \mathrm{H}), 3.83(\mathrm{bs}, 1 \mathrm{H}), 3.58(\mathrm{dm}, J=8.3 \mathrm{~Hz}, 1 \mathrm{H}), 2.31-2.26(\mathrm{~m}, 2 \mathrm{H})$, 2.25-2.22 (m, 2H), $1.65(\mathrm{bs}, 3 \mathrm{H}), 1.11(\mathrm{~s}, 3 \mathrm{H}), 1.08(\mathrm{~s}, 3 \mathrm{H})$.

Cyclopenta[b]furan 3d. ${ }^{7}$ Yield $135 \mathrm{mg}$ (66\%). Colorless liquid. ${ }^{1} \mathrm{H}$ NMR (300 MHz, $\left.\mathrm{CDCl}_{3}\right): \delta 6.09-6.01(\mathrm{~m}, 1 \mathrm{H}), 5.84-5.74(\mathrm{~m}, 2 \mathrm{H}), 3.34(\mathrm{dm}, J=8.4 \mathrm{~Hz}, 1 \mathrm{H}), 2.81-2.71(\mathrm{~m}$, 1H), 2.24 (bs, 2H), 2.19 (bs, 2H), 1.67-1.38 (m, 2H), 1.08 (bs, 3H), 1.06 (bs, 3H), 0.96 (t, J $=7.3 \mathrm{~Hz}, 3 \mathrm{H})$.

Cyclopenta[b]furan $3 \boldsymbol{e} .{ }^{7}$ Yield $191 \mathrm{mg}(64 \%)$. Colorless to pale yellow liquid. ${ }^{1} \mathrm{H}$ NMR $\left(300 \mathrm{MHz}, \mathrm{CDCl}_{3}\right): \delta 6.00(\mathrm{~d}, J=2.9 \mathrm{~Hz}, 1 \mathrm{H}), 5.89(\mathrm{dm}, J=8.2 \mathrm{~Hz}, 1 \mathrm{H}), 5.85(\mathrm{~d}, J=2.2$ 
$\mathrm{Hz}, 1 \mathrm{H}), 5.58-5.54(\mathrm{~m}, 1 \mathrm{H}), 3.84(\mathrm{bs}, 1 \mathrm{H}), 3.78(\mathrm{bd}, J=8.3 \mathrm{~Hz}, 1 \mathrm{H}), 2.27$ (bs, 2H), 2.252.19 (overlapping signals, 5H), $1.69(\mathrm{~s}, 3 \mathrm{H}), 1.09$ (s, 3H), 1.07 (s, 3H).

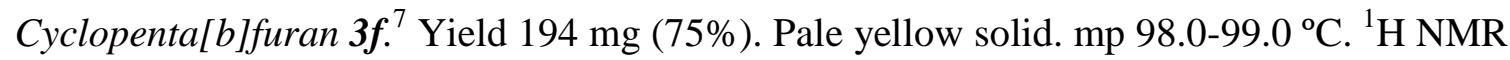
$\left(300 \mathrm{MHz}, \mathrm{CDCl}_{3}\right): \delta 5.72(\mathrm{dtd}, J=8.8 \mathrm{~Hz}, J=2.2 \mathrm{~Hz}, J=0.6 \mathrm{~Hz}, 1 \mathrm{H}), 5.41(\mathrm{q}, J=1.8$ $\mathrm{Hz}, 1 \mathrm{H}), 3.31(\mathrm{dq}, J=8.8 \mathrm{~Hz}, J=1.8 \mathrm{~Hz}, 1 \mathrm{H}), 2.50(\mathrm{dm}, J=13.2 \mathrm{~Hz}, 1 \mathrm{H}), 2.42-2.31(\mathrm{~m}$, $2 \mathrm{H}), 2.25(\mathrm{~d}, J=1.3 \mathrm{~Hz}, 2 \mathrm{H}), 2.20(\mathrm{~s}, 2 \mathrm{H}), 1.97(\mathrm{btd}, J=13.2 \mathrm{~Hz}, J=5.4 \mathrm{~Hz}, 1 \mathrm{H})$, 1.90-1.79 (m, 1H), 1.79-1.69 (m, 1H), $1.37(\mathrm{qt}, J=13.3 \mathrm{~Hz}, J=3.3 \mathrm{~Hz}, 1 \mathrm{H}), 1.17$ (qt, $J=$ $12.9 \mathrm{~Hz}, J=3.8 \mathrm{~Hz}, 1 \mathrm{H}), 1.08(\mathrm{~s}, 3 \mathrm{H}), 1.07(\mathrm{~s}, 3 \mathrm{H}), 0.98(\mathrm{qd}, J=13.1 \mathrm{~Hz}, J=3.4 \mathrm{~Hz}, 1 \mathrm{H})$. Cyclopenta[b]furan $3 g{ }^{7}$ Yield $109 \mathrm{mg}$ (40\%). Colorless to pale yellow solid. mp 42.0-43.0 ${ }^{\circ} \mathrm{C} .{ }^{1} \mathrm{H} \mathrm{NMR}\left(300 \mathrm{MHz}, \mathrm{CDCl}_{3}\right): \delta 6.02(\mathrm{dd}, J=5.6 \mathrm{~Hz}, J=2.2 \mathrm{~Hz}, 1 \mathrm{H}), 5.84(\mathrm{dt}, J=8.5$ $\mathrm{Hz}, J=2.0 \mathrm{~Hz}, 1 \mathrm{H}), 5.74(\mathrm{dt}, J=5.6 \mathrm{~Hz}, \mathrm{~J}=2.0 \mathrm{~Hz}, 1 \mathrm{H}), 3.29(\mathrm{dq}, J=8.5 \mathrm{~Hz}, J=1.8 \mathrm{~Hz}$, 1H), 2.84 (qquint, $J=7.1 \mathrm{~Hz}, J=2.2 \mathrm{~Hz}, 1 \mathrm{H}), 2.24(\mathrm{~d}, J=1.6 \mathrm{~Hz}, 2 \mathrm{H}), 2.20(\mathrm{bs}, 2 \mathrm{H}), 1.18$ (d, $J=7.3 \mathrm{~Hz}, 3 \mathrm{H}), 1.08(\mathrm{~s}, 3 \mathrm{H}), 1.06(\mathrm{~s}, 3 \mathrm{H})$.

Cyclopenta[b]furan $3 \boldsymbol{h}$. Yield $171 \mathrm{mg}$ (55\%). Colorless to pale yellow liquid. IR (film) $\left(\mathrm{cm}^{-1}\right): 3024,2918,1665,1614,1589,1377,1217 .{ }^{1} \mathrm{H}$ NMR $\left(\mathrm{CDCl}_{3}, 300 \mathrm{MHz}\right): \delta 7.36-$ $7.27(\mathrm{~m}, 2 \mathrm{H}), 7.25-7.18(\mathrm{~m}, 1 \mathrm{H}), 7.18-7.11(\mathrm{~m}, 2 \mathrm{H}), 5.75(\mathrm{dm}, J=8.7 \mathrm{~Hz}, 1 \mathrm{H}), 5.66-5.61$ (m, 1H), $3.76(\mathrm{dm}, J=8.7 \mathrm{~Hz}, 1 \mathrm{H}), 3.67(\mathrm{bs}, 1 \mathrm{H}), 2.23(\mathrm{bd}, J=1.2 \mathrm{~Hz}, 3 \mathrm{H}), 2.18(\mathrm{~s}, 3 \mathrm{H})$, 1.58 (bs, 3H). ${ }^{13} \mathrm{C} \mathrm{NMR}\left(\mathrm{CDCl}_{3}, 75 \mathrm{MHz}\right): \delta 194.4(\mathrm{C}), 167.1(\mathrm{C}), 150.1(\mathrm{C}), 143.1(\mathrm{C})$, $128.6(2 \times \mathrm{CH}), 127.3(2 \times \mathrm{CH}), 126.5(\mathrm{CH}), 124.3(\mathrm{CH}), 116.9(\mathrm{C}), 91.5(\mathrm{CH}), 63.0(\mathrm{CH})$, $55.8(\mathrm{CH}), 29.6\left(\mathrm{CH}_{3}\right), 15.3\left(\mathrm{CH}_{3}\right), 15.2\left(\mathrm{CH}_{3}\right)$. HRMS (ESI) $\mathrm{m} / z$ calcd. for $\mathrm{C}_{17} \mathrm{H}_{18} \mathrm{O}_{2} \mathrm{Na}[\mathrm{M}$ $+\mathrm{Na}]^{+} 277.1199$, found 277.1191 . 


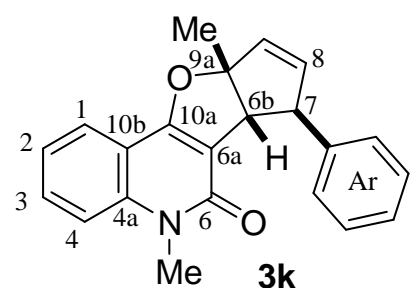

Cyclopenta[b]furan 3k. Yield $204 \mathrm{mg}$ (62\%). Pale yellow solid. mp 119.0-120.0 ${ }^{\circ} \mathrm{C}$. IR (film) $\left(\mathrm{cm}^{-1}\right): 3057,3026,2970,2924,1655,1636,1595,1354,1094 .{ }^{1} \mathrm{H}$ NMR $\left(\mathrm{CDCl}_{3}\right.$, $300 \mathrm{MHz}): \delta 7.78(\mathrm{dd}, J=7.8 \mathrm{~Hz}, J=1.3 \mathrm{~Hz}, 1 \mathrm{H}, 1-\mathrm{H}), 7.57(\mathrm{ddd}, J=8.6 \mathrm{~Hz}, J=7.6 \mathrm{~Hz}$, $J=1.4 \mathrm{~Hz}, 1 \mathrm{H}, 3-\mathrm{H}), 7.50-7.43(\mathrm{~m}, 2 \mathrm{H}, \mathrm{Ar}-\mathrm{H}), 7.41-7.31$ (overlapping m, 3H, 4-H, Ar-H), 7.28-7.18 (overlapping m, 2H, 2-H, Ar-H), 6.11-6.04 (m, 2H, 8-H, 9-H), 4.33 (bs, 1H, 7$\mathrm{H}), 3.73\left(\mathrm{~s}, 3 \mathrm{H}, \mathrm{N}-\mathrm{CH}_{3}\right), 3.55(\mathrm{bd}, J=1.7 \mathrm{~Hz}, 1 \mathrm{H}, 6 \mathrm{~b}-\mathrm{H}), 1.71\left(\mathrm{~s}, 3 \mathrm{H}, 9 \mathrm{a}-\mathrm{CH}_{3}\right) .{ }^{13} \mathrm{C} \mathrm{NMR}$ $\left(\mathrm{CDCl}_{3}, 75 \mathrm{MHz}\right): \delta 161.3(\mathrm{C}, \mathrm{C}-6), 160.0$ (C, C-10a), 143.6 (C, Ar), 140.6 (C, C-4a), $137.1(\mathrm{CH}, \mathrm{C}-8), 133.8(\mathrm{CH}, \mathrm{C}-9), 130.8(\mathrm{CH}, \mathrm{C}-3), 128.5(2 \times \mathrm{CH}, \mathrm{Ar}), 127.3(2 \times \mathrm{CH}$, Ar), 126.5 (CH, Ar), 123.4 (CH, C-1), $121.4(\mathrm{CH}, \mathrm{C}-2), 114.3$ (CH, C-4), 112.9 (C, C-10b), $111.4(\mathrm{C}, \mathrm{C}-6 \mathrm{a}), 104.3$ (C, C-9a), $58.8(\mathrm{CH}, \mathrm{C}-6 \mathrm{~b}), 56.5(\mathrm{CH}, \mathrm{C}-7), 28.8\left(\mathrm{CH}_{3}, \mathrm{~N}-\mathrm{CH}_{3}\right)$, $25.5\left(\mathrm{CH}_{3}, \mathrm{C} 9 \mathrm{a}-\mathrm{CH}_{3}\right)$. HRMS (ESI) $m / z$ calcd. for $\mathrm{C}_{22} \mathrm{H}_{20} \mathrm{NO}_{2}[\mathrm{M}+\mathrm{H}]^{+} 330.1489$, found 330.1479.

Tandem condensation/hydrogenation for the trapping of $2 \mathrm{H}$-pyran intermediate en route to cyclopenta[b]furans. A mixture of 1,3-dicarbonyl substrate 4 (140 mg, $1 \mathrm{mmol}$ ), unsaturated aldehyde 6 (234 mg, $1 \mathrm{mmol})$, and EDDA (36.0 mg, $0.2 \mathrm{mmol})$ in $\mathrm{CH}_{2} \mathrm{Cl}_{2}(5.0$ $\mathrm{mL}$ ) was heated at reflux for 4 hours. The solvent was then evaporated under reduced pressure and the residue was passed through a short path of silica gel (eluted with hexanes/ethyl acetate). The solvent was then evaporated under reduced pressure and the crude mixture was dissolved in ethyl acetate $(50 \mathrm{ml})$. To this solution was added $\mathrm{PtO}_{2}(30$ $\mathrm{mg}$ ), and the resulting suspension was degassed three times (three vacuum/hydrogen cycles to remove air). The suspension was vigorously stirred under a hydrogen atmosphere 
(balloon, ca.1 atm) at room temperature for 30 minutes, filtered through celite, and concentrated under reduced pressure. The residue was purified by flash column chromatography on silica gel (hexanes/ethyl acetate) to afford products $\mathbf{2} \mathbf{j}_{\text {red }}$ (36 $\mathbf{m g}, \mathbf{1 0 \%}$ yield) and $\mathbf{3} \mathbf{j}_{\text {red }}(197 \mathrm{mg}, 55 \%$ yield).

2H-Pyran $2 j_{\text {red }}$. Colorless solid. mp 140.0-141.0 ${ }^{\circ} \mathrm{C}$. IR (KBr) $\left(\mathrm{cm}^{-1}\right): 3082,3059,3022$, 2951, 2926, 2907, 1637, 1491, 1447, 1398, 1213, 1140. ${ }^{1} \mathrm{H}$ NMR $\left(\mathrm{CDCl}_{3}, 300 \mathrm{MHz}\right): \delta$ 7.36-7.16 (overlapping m, 10H), 3.78 (bs, $1 \mathrm{H}), 3.48$ (bd, $J=6.9 \mathrm{~Hz}, 1 \mathrm{H}), 2.46-2.39$ (overlapping m, 4H), 2.32-2.05 (overlapping m, 4H), $1.16(\mathrm{~s}, 3 \mathrm{H}), 1.12(\mathrm{~s}, 3 \mathrm{H}) .{ }^{13} \mathrm{C} \mathrm{NMR}$ $\left(\mathrm{CDCl}_{3}, 75 \mathrm{MHz}\right): \delta 194.2(\mathrm{C}), 174.8(\mathrm{C}), 144.0(\mathrm{C}), 142.5(\mathrm{C}), 128.4(2 \times \mathrm{CH}), 128.3(2 \times$ $\mathrm{CH}), 127.6(\mathrm{CH}), 127.0(2 \times \mathrm{CH}), 125.8(\mathrm{CH}), 124.7(2 \times \mathrm{CH}), 115.1(\mathrm{C}), 103.8(\mathrm{C}), 59.4$ (CH), $51.0\left(\mathrm{CH}_{2}\right), 50.4(\mathrm{CH}), 39.9\left(\mathrm{CH}_{2}\right), 37.7\left(\mathrm{CH}_{2}\right), 34.1(\mathrm{C}), 29.8\left(\mathrm{CH}_{2}\right), 29.0\left(\mathrm{CH}_{3}\right)$, 28.0 $\left(\mathrm{CH}_{3}\right)$. HRMS (ESI) $\mathrm{m} / z$ calcd. for $\mathrm{C}_{25} \mathrm{H}_{26} \mathrm{NaO}_{2}[\mathrm{M}+\mathrm{Na}]^{+} 381.1825$, found 381.1815. Cyclopenta[b]furan 3j. $\mathbf{j}_{\text {red }}$ Colorless liquid. IR (film) ( $\mathrm{cm}^{-1}$ ): 3059, 3026, 2953, 2926, 2907, 1625, 1392, 1217. ${ }^{1} \mathrm{H}$ NMR $\left(\mathrm{CDCl}_{3}, 300 \mathrm{MHz}\right): \delta 7.35-7.11$ (overlapping $\left.\mathrm{m}, 8 \mathrm{H}\right), 7.10$ 7.02 (overlapping m, 2H), $4.29(\mathrm{dt}, J=10.0 \mathrm{~Hz}, J=3.5 \mathrm{~Hz}, 1 \mathrm{H}), 3.20(\mathrm{td}, J=7.0 \mathrm{~Hz}, J=$ 3.5 Hz, 1H), 2.81-2.45 (overlapping m, 4H), 2.43-2.22 (overlapping m, 4H), 1.84-1.53 (m, 2H), $1.11(\mathrm{~s}, 6 \mathrm{H}) .{ }^{13} \mathrm{C} \mathrm{NMR}\left(\mathrm{CDCl}_{3}, 75 \mathrm{MHz}\right): \delta 197.7(\mathrm{C}), 168.8(\mathrm{C}), 141.1(\mathrm{C}), 139.9$ (C), $128.4(2 \times \mathrm{CH}), 128.28(2 \times \mathrm{CH}), 128.26(2 \times \mathrm{CH}), 127.9(2 \times \mathrm{CH}), 126.7(\mathrm{CH}), 125.9$ $(\mathrm{CH}), 109.7(\mathrm{C}), 79.2(\mathrm{CH}), 50.5\left(\mathrm{CH}_{2}\right), 42.3\left(\mathrm{CH}_{2}\right), 40.2(\mathrm{CH}), 32.1(\mathrm{C}), 32.0\left(\mathrm{CH}_{2}\right), 31.0$ $\left(\mathrm{CH}_{2}\right), 28.5\left(\mathrm{CH}_{3}\right), 28.1\left(\mathrm{CH}_{3}\right), 21.4\left(\mathrm{CH}_{2}\right)$. HRMS (ESI) $\mathrm{m} / z$ calcd. for $\mathrm{C}_{25} \mathrm{H}_{28} \mathrm{NaO}_{2}[\mathrm{M}+$ $\mathrm{Na}]^{+}$383.1981, found 383.1969.

Lewis acid promoted cycloisomerization of $\mathbf{2 H}$-pyran $2 \mathrm{k}$. To a solution of pyran derivative $2 \mathbf{k}^{6 \mathrm{a}}$ (329 mg, $\left.1 \mathrm{mmol}\right)$ in $\mathrm{CH}_{2} \mathrm{Cl}_{2}(10.0 \mathrm{ml}, 0.1 \mathrm{M}), \mathrm{FeCl}_{3}(167 \mathrm{mg}, 1 \mathrm{mmol})$ was 
added. The mixture was heated at reflux until complete consumption of substrate (TLC monitoring, approximately 3 hours). The solvent was then evaporated under reduced pressure and the residue was purified by flash column chromatography on silica gel (hexanes/ethyl acetate) to afford cyclopenta[b]furan $\mathbf{3 k}$ (132 mg, $40 \%$ yield).

\section{ACKNOWLEDGEMENTS}

We thank Argentina-France MINCyT-ECOS 2013 (project A13E06) for financial support. We also thank Universidad Nacional de Rosario, Fundación Josefina Prats and CONICET for financial support. M.J.R. thanks CONICET for fellowships. We are grateful to Dr. G.R. Labadie and Dr. C.M.J. Delpiccolo for HRMS measurements. V.G. thanks UPS, IUF, and CNRS. We used the computing facility of the CRIHAN (project 2006-013).

\section{SUPPORTING INFORMATION}

Copies of ${ }^{1} \mathrm{H}$ NMR spectra of known compounds prepared by new methodology, and ${ }^{1} \mathrm{H}$, ${ }^{13} \mathrm{C}$ and $2 \mathrm{D}$ NMR spectra for all new compounds. This material is available free of charge via the Internet at http://pubs.acs.org.

\section{REFERENCES AND FOOTNOTES}

(1) For a review on biosynthetic and biomimetic electrocyclizations, see: (a) Beaudry, C. M.; Malerich, J. P.; Trauner, D. Chem. Rev. 2005, 105, 4757, and references cited therein. The interested reader should also consult: (b) Biomimetic Organic Synthesis, 2 Vols.; Poupon, E.; Nay, B., Eds.; Wiley-VCH Verlag, Weinheim, 2011. (c) Miller, A. K.; Trauner, D. Synlett 2006, 2295. (d) Moses, J. E.; Baldwin, J. E.; Brückner, S.; Eade, S. J.; Adlington, R. M. Org. Biomol. Chem. 2003, 1, 3670. 
(2) For reviews on transition metal-catalyzed and/or electrophile-promoted cycloisomerizations, see: (a) Yamamoto, Y. Chem. Rev. 2012, 112, 4736. (b) Aubert, C.; Fensterbank, L.; Garcia, P.; Malacria, M.; Simonneau, A. Chem. Rev. 2011, 111, 1954. (c) Fürstner, A. Chem. Soc. Rev. 2009, 38, 3208. (d) Yoder, R. A.; Johnston, J. N. Chem. Rev. 2005, 105, 4730. (e) Eschenmoser, A.; Arigoni, D. Helv. Chim. Acta 2005, 88, 3011. (f) Ojima, I.; Tzamarioudaki, M.; Li, Z.; Donovan, R. J. Chem. Rev. 1996, 96, 635.

(3) For a recent review on catalytic enantioselective cycloisomerizations, see: Watson, I. D. G.; Toste, F. D. Chem. Sci. 2012, 3, 2899.

(4) (a) Riveira, M. J.; Mischne, M. P. Synth. Commun. 2013, 43, 208. (b) Hsung, R. P.; Kurdyumov, A. V.; Sydorenko, N. Eur. J. Org. Chem. 2005, 23, and references cited therein.

(5) For a recent review on domino reactions based on Knoevenagel condensations, see: Voskressensky, L. G.; Festa, A. A.; Varlamov, A. V. Tetrahedron 2014, 70, 551.

(6) (a) Riveira, M. J.; Mischne, M. P. Chem.-Eur. J. 2012, 18, 2382. (b) Riveira, M. J.; Gayathri, C.; Navarro-Vázquez, A.; Tsarevsky, N. V.; Gil, R. R.; Mischne, M. P. Org. Biomol. Chem. 2011, 9, 3170.

(7) Riveira, M. J.; Mischne, M. P. J. Org. Chem. 2014, 79, 8244.

(8) Spirochromenes comprise the most important class of photochromic $2 H$-pyrans.

(9) (a) Buback, J.; Kullmann, M.; Langhojer, F.; Nuernberger, P.; Schmidt, R.; Würthner, F.; Brixner, T. J. Am. Chem. Soc. 2010, 132, 16510. (b) Mandal, S.; Parida, K. N.; Samanta, S.; Moorthy, J. N. J. Org. Chem. 2011, 76, 7406. (c) Huang, C.-N.; Kuo, P.-Y.; Lin, C.-H.; Yang, D.-Y. Tetrahedron 2007, 63, 10025. For the first report on the isomerization of a 
dienone to an $\alpha$-pyran, see: (d) Marvell, E. N.; Caple, G.; Gosink, T. A.; Zimmer, G. J. Am. Chem. Soc. 1966, 88, 619.

(10) Krasnaya, Z. A. Chem. Heterocycl. Comp. 1999, 35, 1255.

(11) Shen, H. C.; Wang, J.; Cole, K. P.; McLaughlin, M. J.; Morgan, C. D.; Douglas, C. J.; Hsung, R. P.; Coverdale, H. A.; Gerasyuto, A. I.; Hahn, J. M.; Liu, J.; Sklenicka, H. M.; Wei, L.-L.; Zehnder, L. R.; Zificsak, C. A. J. Org. Chem. 2003, 68, 1729.

(12) Products $\mathbf{3 c}$ and $\mathbf{3 f}$ could not be previously obtained neither using ferric chloride as catalyst nor a Brønsted acid such as camphorsulfonic acid; they undergo further rearrangement to a different isomer. See Ref. 7.

(13) (a) Álvarez, R.; Vaz, B.; Gronemeyer, H.; de Lera, A. R. Chem. Rev. 2014, 114, 1. (b) Zhu, Y.; Ganapathy, S.; Liu, R. S. H. J. Org. Chem. 1992, 57, 1110. (c) Kluge, A. F.; Lillya, C. P. J. Org. Chem. 1971, 36, 1977. (d) Kluge, A. F.; Lillya, C. P. J. Org. Chem. 1971, 36, 1988.

(14) Gaussian 09, Revision D.01, Frisch, M. J. et al., Gaussian, Inc., Wallingford CT, 2009.

(15) For previous examples on the hydrogenation of $2 \mathrm{H}$-pyrans obtained via Knoevenagel condensation between $\beta$-ketoesteres and $\alpha, \beta$-unsaturated aldehydes, see: Peng, W.; Hirabaru, T.; Kawafuchi, H.; Inokuchi, T. Eur. J. Org. Chem. 2011, 5469.

(16) (a) Ribeiro, N.; Thuaud, F.; Nebigil, C.; Désaubry, L. Bioorg. Med. Chem. 2012, 20, 1857, and references therein. (b) Magnus, P.; Freund, W. A.; Moorhead, E. J.; Rainey, T. J. Am. Chem. Soc. 2012, 134, 6140. (c) Fletcher, C. J.; Blair, D. J.; Wheelhouse, K. M. P.; Aggarwal, V. K. Tetrahedron 2012, 68, 7598. (d) Li, N.; Di, L.; Gao, W.-C.; Wang, K.-J.; Zu, L.-B. J. Nat. Prod. 2012, 75, 1723. (e) Malona, J. A.; Cariou, K.; Frontier, A. J. J. Am. Chem. Soc. 2009, 131, 7560. (f) Enomoto, M.; Kuwahara, S. J. Org. Chem. 2006, 71, 6287. 
(g) Che, Y.; Araujo, A. R.; Gloer, J. B.; Scott, J. A.; Malloch, D. J. Nat. Prod. 2005, 68, 435.

(h) Gerard, B.; Jones II, G.; Porco, J. A. Jr. J. Am. Chem. Soc. 2004, 126, 13620. (i) Larock, R. C.; Lee, N. H. J. Org. Chem. 1991, 56, 6253.

(17) (a) Miyagawa, T.; Nagai, K.; Yamada, A.; Sugihara, Y.; Fukuda, T.; Fukuda, T.; Uchida, R.; Tomoda, H.; Õmura, S.; Nagamitsu, T. Org. Lett. 2011, 13, 1158. (b) Fotiadou, A. D.; Zografos, A. L. Org. Lett. 2011, 13, 4592. 